\title{
Generalized column distances for convolutional codes
}

\author{
Sara D. Cardell \\ Instituto de Matemática, Estatística \\ e Computação Científica \\ Universidade Estadual de Campinas, Brasi \\ Email: sdcardell@ime.unicamp.br
}

Marcelo Firer

Instituto de Matemática, Estatística

e Computação Científica

Universidade Estadual de Campinas, Brasil

Email: mfirer@ime.unicamp.br

\author{
Diego Napp \\ Departamento de Matemática, \\ Universidade de Aveiro, Portugal \\ Email: diego@ua.pt
}

\begin{abstract}
In this work, we adapt the notion of generalized Hamming weight of block codes to introduce the novel concept of generalized column distances for convolutional codes. This can be considered as an extension of the work done in [18] on the generalized Hamming weights for free distance of convolutional codes. We also introduce the concept of Almost-MDP and NearMDP convolutional code. The problem of constructing convolutional codes with design generalized column distances remains an interesting open problem that requires further research.
\end{abstract}

\section{INTRODUCTION}

In [1], Wei introduced the concept of generalized Hamming weights (GHW) and the Hamming weight hierarchy for linear block codes $(\mathrm{HWH})$. Let $\mathcal{C}$ be an $[n, k]$ linear code over a Galois field $\mathbb{F}$. We define the support of $\mathcal{C}$ as

$$
\operatorname{supp}(\mathcal{C})=\left\{i \mid x_{i} \neq 0 \text { for some }\left[x_{1}, x_{2}, \ldots, x_{n}\right] \in \mathcal{C}\right\}
$$

The $r$-th generalized Hamming weight of $\mathcal{C}$ is then defined as

$$
\begin{aligned}
& d_{r}(\mathcal{C})=\min \{|\operatorname{supp}(\mathcal{D})| \mid \\
&\mathcal{D} \text { is an } r-\text { dimensional subcode of } \mathcal{C}\},
\end{aligned}
$$

and the HWH is the set of integers $\left\{d_{r}(\mathcal{C}) \mid 1 \leq r \leq k\right\}$.

Although the GHW were originally introduced motivated by applications in cryptography in [1], [9], it was Forney that explicitly showed in [8] the close and deep connections between the Hamming weight hierarchy (also called length/dimension profile) and the trellis complexity. The GHW have, since then, generated notable interest among coding theorists.

It was observed in [8] that the results obtained for block codes could be extended to convolutional codes. This motivated the work in [18] where the convolutional case was addressed. In this case a convolutional code $\mathcal{C}$ was seen as an infinite-dimensional linear $\mathbb{F}$-vector space and therefore $d_{r}(\mathcal{C})$ was well-defined for all $r \in \mathbb{N}_{0}$. In this contribution we aim at continuing this line of research by further investigating the GHW in the context of convolutional codes. In particular, we focus on column distances instead of on the free distance and propose a definition of generalized column distance (GCD) for convolutional codes. As we will see, most of the properties of the GHW for block codes apply also for this notion of column distances of convolutional codes.
Column distances of convolutional codes over finite fields have been already studied for decades [20]. However, the notion of Maximum Distance Profile (MDP) convolutional codes over (non-binary) finite fields have been defined and fully studied by Rosenthal et al. in [11], [17]. These codes, that are closely related to convolutional codes with optimum distance profile (ODP) [20, p. 112], are characterized by the property that their column distances increase as rapidly as possible for as long as possible. Obviously, fast growth of the column distances is an important property for codes to be used with sequential decoding and therefore very appealing for applications (see [10]). Indeed, the maximal possible growth in the column distances means that these codes have the potential to correct the maximal number of errors per time interval. However, the problem of building MDP convolutional codes over small finite fields is still open and seems highly nontrivial (if possible at all). The two existing general constructions of MDP convolutional codes (see [15], [16], [17]) require far too large finite fields. Hence, one approach to this problem is to relax the MDP condition and try to find convolutional codes that are not MDP but posses good design column distance profile over small fields. To this end, we extend the notions of Almost-Maximal Distance Separable (MDS) and Near-MDS to the notions of $\alpha$-Almost-Maximum Distance Profile (MDP) and $s$-Near- MDP. Moreover, considering the generalized weights, we introduce a refined figure of merit of a code to be MDP: the matrix of defects and its norm. We will present an example of a 3-Near-MDP convolutional code of length 4 , dimension 2 and degree 2 over $\mathbb{F}_{3}$. The minimum field size for constructing an MDP convolutional code with these parameters is 17 .

Finally, we indicate the possibility of considering yet another notion of GCD (see Remark 2). It is not clear whether these two are equivalent indicators of the performance of convolutional codes within a time interval. This issue lies at the core of the problem of extending the notion of the GHW from block codes to GCD and requires further investigation.

The rest of the paper is organized as follows: In Section II, we recall basic notions of convolutional codes. In Section III we introduce the notion of generalized column distance of convolutional codes. The remaining sections are devoted to derive the main properties of these distances and to introduce the new 
concepts of $\alpha$-Almost-MDP and $s$-Near-MDP. We conclude the paper by presenting an example of a convolutional code that has good column distance profile over $\mathbb{F}_{3}$ together with some conclusions.

\section{Convolutional CODES}

Let $\mathbb{F}$ be a finite field and $\mathbb{F}[D]$, the ring of polynomials with coefficients in $\mathbb{F}$. A convolutional code $\mathcal{C}$ of rate $k / n$ is an $\mathbb{F}[D]$-module of $\mathbb{F}[D]^{n}$ of rank $k$ of the form

$$
\mathcal{C}=\operatorname{im}_{\mathbb{F}[D]} G(D)=\left\{u(D) G(D) \mid u(D) \in \mathbb{F}^{k}[D]\right\}
$$

where $G(D) \in \mathbb{F}[D]^{k \times n}$ is a right invertible matrix or basic, i.e., there exits a matrix $H(D) \in \mathbb{F}[D]^{(n-k) \times n}$ such that

$$
\operatorname{im}_{\mathbb{F}[D]} G(D)=\operatorname{ker}_{\mathbb{F}[D]} H(D)=\left\{v \in \mathbb{F}[D]^{n} \mid v H^{T}=0\right\} .
$$

The degree $\delta$ of $\mathcal{C}$ is defined as the maximum degree of the full size minors of $G(D)$.

As we know, the most important property of a code is its distance. In this case, we define the weight of a polynomial vector $v(D)=\sum_{i} \boldsymbol{v}_{i} D^{i} \in \mathbb{F}[D]^{n}$, with $\boldsymbol{v}_{i} \in \mathbb{F}^{n}$, as

$$
\omega(v(D))=\sum_{i} \omega\left(\boldsymbol{v}_{i}\right)
$$

where $\omega\left(\boldsymbol{v}_{i}\right)$ is the Hamming weight of $\boldsymbol{v}_{i}$. The free distance is, thus, defined as

$$
d(\mathcal{C})=\min \{\omega(v(D)) \mid v(D) \in \mathcal{C}, v(D) \neq 0\} .
$$

We can express the generator matrix as

$$
G(D)=\sum_{j=0}^{\mu} G_{j} D^{j}, G_{j} \in \mathbb{F}^{k \times n}, G_{\mu} \neq 0
$$

and the parity-check matrix as

$$
H(D)=\sum_{j=0}^{\nu} H_{j} D^{j}, H_{j} \in \mathbb{F}^{(n-k) \times n}, H_{\nu} \neq 0 .
$$

Let

$$
G_{j}^{c}=\left[\begin{array}{cccc}
G_{0} & G_{1} & \cdots & G_{j} \\
0 & G_{0} & \cdots & G_{j-1} \\
\vdots & \vdots & \cdots & \vdots \\
0 & 0 & \cdots & G_{0}
\end{array}\right]
$$

and

$$
H_{j}^{c}=\left[\begin{array}{cccc}
H_{0} & 0 & \cdots & 0 \\
H_{1} & H_{0} & \cdots & 0 \\
\vdots & \vdots & \cdots & \vdots \\
H_{j} & H_{j-1} & \cdots & H_{0}
\end{array}\right]
$$

be the truncated sliding generator and parity-check matrices for $j \in \mathbb{N}_{0}$, respectively.

Notice that each codeword of $\mathcal{C}$ has the form $v(D)=$ $\sum_{i} \boldsymbol{v}_{i} D^{i}$, with $\boldsymbol{v}_{i} \in \mathbb{F}_{q}^{n}$, and it can be seen as a vector $\boldsymbol{v}=\left[\boldsymbol{v}_{0}, \boldsymbol{v}_{1}, \boldsymbol{v}_{2}, \ldots\right]$.

Now, we can define the $\mathbf{j}$-th column distance [20] as

$$
d_{j}(\mathcal{C})=\min \left\{\omega\left(\left[\mathbf{u}_{0}, \mathbf{u}_{1}, \ldots, \mathbf{u}_{j}\right] G_{j}^{c}\right) \mid \mathbf{u}_{i} \in \mathbb{F}^{k}, \mathbf{u}_{0} \neq 0\right\} .
$$

For simplicity we will use $d_{j}$ instead of $d_{j}(\mathcal{C})$. We remark that since the generator matrix is basic, the column distance is a property of the code $\mathcal{C}$ and does not depend on the choice of the generator matrix [17], [20].

Convolutional codes with large column distance are very appealing for sequential decoding. The larger the column distance is, the more number of errors can be corrected per time interval. Maximum Distance Profile (MDP) convolutional codes were introduced in [17] and are basically convolutional codes characterized by the property that their initial column distances increase as rapidly as possible for as long as possible, i.e., they are convolutional codes that are optimal with respect to the column distance. Formally speaking, a convolutional code $\mathcal{C}$ of rate $k / n$ and degree $\delta$, is MDP if

$$
d_{j}=(n-k)(j+1)+1 \text { for } j=1,2, \ldots, L,
$$

where $L=\lfloor\delta / k\rfloor+\lfloor\delta /(n-k)\rfloor$. Given a convolutional code $\mathcal{C}$, the set of integers $\left\{d_{j} \mid 1 \leq j \leq L\right\}$ is called the column distance profile of $\mathcal{C}$.

\section{Generalized Column Distances of CONVOLUTIONAL CODES}

In order to generalized the column distances, we define

$$
\mathcal{C}_{j}=\left\{\left[\mathbf{u}_{0}, \mathbf{u}_{1}, \ldots, \mathbf{u}_{j}\right] G_{j}^{c} \mid \mathbf{u}_{i} \in \mathbb{F}^{k}, \mathbf{u}_{0} \neq 0\right\} .
$$

Note that $\mathcal{C}_{j}$ does not depend on the choice of the generator matrix $G$, since the elements of $\mathcal{C}_{j}$ are truncated codewords of $\mathcal{C}$.

Notation: If $\boldsymbol{v}^{i} \in \mathcal{C}_{j} \subset \mathbb{F}^{n(j+1)}$, we write $\boldsymbol{v}^{i}=\left[\boldsymbol{v}_{0}^{i}, \ldots, \boldsymbol{v}_{j}^{i}\right]$ with $\boldsymbol{v}_{k}^{i}=\left[v_{1, k}^{i}, \ldots, v_{n, k}^{i}\right] \in \mathbb{F}^{n}$ for $k=0, \ldots, j$. Denote by $<\boldsymbol{v}^{1}, \ldots, \boldsymbol{v}^{r}>_{\mathbb{F}}$ the $\mathbb{F}$-vector subspace spanned by $\left\{\boldsymbol{v}^{1}, \ldots, \boldsymbol{v}^{r}\right\}$ over $\mathbb{F}$.

Obviously $\boldsymbol{v}^{i} \in \mathcal{C}_{j}$ implies that $\boldsymbol{v}_{0}^{i} \neq 0$ as $G(0)$ is full row rank $\left(G(D)\right.$ is right invertible). Note that $\left\{\boldsymbol{v}^{1}, \ldots, \boldsymbol{v}^{r}\right\} \subset \mathcal{C}_{j}$ does not implies that $\left\langle\boldsymbol{v}^{1}, \ldots, \boldsymbol{v}^{r}>_{\mathbb{F}} \subset \mathcal{C}_{j}\right.$. In fact, we have that $0 \in<\boldsymbol{v}^{1}, \ldots, \boldsymbol{v}^{r}>_{\mathbb{F}}$ and $0 \notin \mathcal{C}_{j}$.

Let $\mathcal{D}$ be a subset of $\mathcal{C}_{j}$. We define the support of $\mathcal{D}$ as

$$
\operatorname{supp}(\mathcal{D})=\left\{(r, s) \mid v_{r, s}^{i} \neq 0, \text { for some } \boldsymbol{v}^{\boldsymbol{i}} \in \mathcal{D}\right\},
$$

and, then, the weight of $\mathcal{D}$ can be defined as $\omega(\mathcal{D})=$ $|\operatorname{supp}(\mathcal{D})|$, that is, the cardinality of $\operatorname{supp}(\mathcal{D})$.

We are ready now to define the $\boldsymbol{j}$-th Generalized Column Distance (GCD) of $\mathcal{C}$ :

$$
\begin{aligned}
d_{j}^{r}(\mathcal{C})=\min \{\omega(V) \mid & V=<\boldsymbol{v}^{1}, \ldots, \boldsymbol{v}^{r}>_{\mathbb{F}} \backslash\{0\} \subseteq \mathcal{C}_{j}, \\
& \text { where } \left.\left\{\boldsymbol{v}_{0}^{1}, \cdots, \boldsymbol{v}_{0}^{r}\right\} \text { is a L.I. set }\right\},
\end{aligned}
$$

where L.I. stands for linearly independent. For simplicity we will use $d_{j}^{r}$ instead of $d_{j}^{r}(\mathcal{C})$. Note that $\mathcal{C}$ can be viewed as an infinite-dimensional vector space over $\mathbb{F}$. Since we demand $\left\{\boldsymbol{v}_{0}^{1}, \cdots, \boldsymbol{v}_{0}^{r}\right\}$ to be a L.I. set, we have that $d_{j}^{r}$ is well defined only for $r=1,2, \ldots, k$ and not for all $r \in \mathbb{N}_{0}$ as happens in [18]. In this sense, the situation addressed here is closer to the block code case . We call $\left\{d_{j}^{1}, \ldots, d_{j}^{k}\right\}$ the $j$-th GCD profile 
of $\mathcal{C}$. We remark, however, that the number of different sets of the type $\left\langle\boldsymbol{v}^{1}, \ldots, \boldsymbol{v}^{r}>_{\mathbb{F}} \backslash\{0\}\right.$ considered in (1) is upper bounded by

$$
\left(\begin{array}{c}
k(j+1) \\
r
\end{array}\right)-\left(\begin{array}{c}
k j \\
r
\end{array}\right)
$$

whereas for a $[n, k]$-block code the equivalent number is upper bounded by $\left(\begin{array}{l}k \\ r\end{array}\right)$.

\section{PROPERTIES AND RESUltS}

In the next result we show the monotonicity of the GCD of a convolutional code.

Theorem 1. Let $\mathcal{C}$ be a convolutional code and $\left\{d_{j}^{1}, \ldots, d_{j}^{k}\right\}$ its $j$-th GCD profile. Then,

$$
d_{j}(\mathcal{C})=d_{j}^{1}<\cdots<d_{j}^{k} .
$$

Sketch of the proof. Let $V=<\boldsymbol{v}^{1}, \boldsymbol{v}^{2}, \ldots, \boldsymbol{v}^{r+1}>_{\mathbb{F}} \backslash\{0\} \subset$ $\mathcal{C}_{j}$ be a subset of $\mathcal{C}_{j}$ such that $\omega(V)=d_{j}^{r+1}$. Obviously, there exists $\nu \in\{1, \ldots, n\}$, such that $v_{\nu, 0}^{1} \neq 0$. One can show that performing linear combinations over $\mathbb{F}$ we can obtain that $V=<\boldsymbol{v}^{1}, \widehat{\boldsymbol{v}}^{2}, \ldots, \widehat{\boldsymbol{v}}^{r+1}>_{\mathbb{F}} \subset \mathcal{C}_{j}$ with $\widehat{v}_{\nu, 0}^{i}=0$, for $i=2, \ldots, r+1$. Denote $\widehat{V}_{\text {trunc }}=<\widehat{\boldsymbol{v}}^{2}, \ldots, \widehat{\boldsymbol{v}}^{r+1}>_{\mathbb{F}}$. We know that $(\nu, 0) \in \operatorname{supp}(V)$, but $(\nu, 0) \notin \operatorname{supp}\left(\widehat{V}_{\text {trunc }}\right)$ and this means that $\operatorname{supp}\left(\widehat{V}_{\text {trunc }}\right) \subset \operatorname{supp}(V)$ and the inclusion is strict. Therefore, $\omega\left(\widehat{V}_{\text {trunc }}\right)<\omega(V)$ and $d_{j}^{r}<d_{j}^{r+1}$.

The following straightforward result follows.

Corollary 1. Let $V=<\boldsymbol{v}^{1}, \ldots, \boldsymbol{v}^{r}>_{\mathbb{F}} \backslash\{0\} \subset \mathcal{C}_{j}$ such that $\left\{\boldsymbol{v}_{0}^{1}, \ldots, \boldsymbol{v}_{0}^{r}\right\}$ is a L.I. set and $d_{j}^{r}=\omega(V)$. If

$$
\omega\left(<\boldsymbol{v}^{1}, \ldots, \boldsymbol{v}^{r}>_{\mathbb{F}}\right)=\omega\left(<\boldsymbol{v}^{1}, \ldots, \boldsymbol{v}^{r}, \boldsymbol{v}^{r+1}>_{\mathbb{F}}\right)
$$

then

$$
\boldsymbol{v}_{0}^{r+1} \in<\boldsymbol{v}_{0}^{1}, \ldots, \boldsymbol{v}_{0}^{r}>_{\mathbb{F}}
$$

Proof. If (2) holds and $\left\{\boldsymbol{v}_{0}^{1}, \ldots, \boldsymbol{v}_{0}^{r+1}\right\}$ are L.I., then we would have that $d_{j}^{r}=d_{j}^{r+1}$ which contradicts Theorem 1 . Therefore, we necessarily have that $\left.\boldsymbol{v}_{0}^{r+1} \in<\boldsymbol{v}_{0}^{1}, \ldots, \boldsymbol{v}_{0}^{r}\right\rangle_{\mathbb{F}}$.

Now, we are ready to introduce a bound for our weights.

Proposition 1. Given a convolutional code of rate $k / n$ and degree $\delta$, it holds that

$$
d_{j}^{r} \leq(n-k)(j+1)+r
$$

for $j=0,1, \ldots, L$ and $r=1,2, \ldots, k$.

Proof. Let $G(D)$ be a generator matrix of $\mathcal{C}$. It is easy to check that as $G(0)$ is full row rank we can obtain, after a appropriate permutation of columns (which is an isometry) and elementary row operations, a truncated sliding matrix which has the following form:

$$
\hat{G}_{j}^{c}=\left[\begin{array}{ccccc:ccccc}
I & \mathbf{0} & \mathbf{0} & \cdots & \mathbf{0} & P_{0} & P_{1} & P_{2} & \cdots & P_{j} \\
\mathbf{0} & I & \mathbf{0} & \cdots & \mathbf{0} & \mathbf{0} & P_{0} & P_{1} & \cdots & P_{j-1} \\
\mathbf{0} & \mathbf{0} & I & \cdots & \mathbf{0} & \mathbf{0} & \mathbf{0} & P_{0} & \cdots & P_{j-2} \\
\vdots & \vdots & \vdots & & \vdots & \vdots & \vdots & \vdots & & \vdots \\
\mathbf{0} & \mathbf{0} & \mathbf{0} & \cdots & I & \mathbf{0} & \mathbf{0} & \mathbf{0} & \cdots & P_{0}
\end{array}\right],
$$

from where the result readily follows.

Remark 1. Taking into account the monotonicity of the GCD of a convolutional code (Theorem 1) and Proposition 1, it is easy to see that if one of the $j$-th column distance $d_{j}^{r}$ attains the bound (3) for some $r$, all following generalized column distances $d_{j}^{s}$ will also attain the bound for $s \geq r$.

Remark 2. When defining the $j$-th GCD, we consider subspaces generated by a set $\left\{\boldsymbol{v}^{1}, \cdots, \boldsymbol{v}^{r}\right\}$ with the property that $\left\{\boldsymbol{v}_{0}^{1}, \cdots, \boldsymbol{v}_{0}^{r}\right\}$ is a L.I. set. A relaxation of this condition, requiring $\left\{\boldsymbol{v}^{1}, \cdots, \boldsymbol{v}^{r}\right\}$ to be a L.I. set, would allow us to define the $r$-th generalized weight for every $r \leq(j+1) k$. Although exploring such a possibility is interesting, it is a harder problem, since it can be shown that in this case the generalized column distances need not to be strictly increasing. This would imply that it is possible to have $d_{j}^{r}$ attaining the bound while $d_{j}^{r+1}$ is not maximal.

The following characterization of $d_{j}^{r}$ in terms of $H_{j}^{c}$ follows from Wei's original work [1, Theorem 2] and the fact that for $\boldsymbol{v}^{i} \in \mathcal{C}_{j}$ we require that $\boldsymbol{v}_{0}^{i} \neq 0$.

Theorem 2. Let $\mathcal{C}$ be a convolutional code with a truncated parity-check matrix $H_{j}^{c}$. Then, $\mathcal{C}$ has $j$-th $G C D d_{j}^{r}$ if and only if $d_{j}^{r}$ is the smallest number with the property that there is a set of $d_{j}^{r}$ columns of $H_{j}^{c}$, containing at least one of the first $n$ columns of $H_{j}^{c}$, whose rank is equal to or less than $d_{j}^{r}-r$.

\section{Almost And Near MDP Codes}

As pointed out in [12], [13], [14] the generalized weights refine the minimal distance as a figure of merit for codes. In this section we follow this idea and establish how far a code is from attaining the bounds for the generalized column distances. To be explicit, let us consider the bound given in Proposition 1 for $d_{j}^{r}$ and denote it by: $\Omega_{j}^{r}=(n-k)(j+1)+r$. We can define the defect of $d_{j}^{r}$ as $\Lambda_{j}^{r}=d_{j}^{r}-\Omega_{j}^{r}$ and the matrix of defects of a code as the matrix given by:

$$
\boldsymbol{\Lambda}=\left[\begin{array}{cccc}
\Lambda_{0}^{1} & \Lambda_{0}^{2} & & \Lambda_{0}^{k} \\
\Lambda_{1}^{1} & \Lambda_{1}^{2} & \cdots & \Lambda_{1}^{k} \\
\vdots & \vdots & \cdots & \vdots \\
\Lambda_{L}^{1} & \Lambda_{L}^{2} & \cdots & \Lambda_{L}^{k}
\end{array}\right]
$$

In terms of the defect, to say that a code $\mathcal{C}$ is MDP means that $\Lambda_{j}^{1}=0$, for all $j=0,1, \ldots, L$, i.e., the first column, and therefore the whole matrix, is zero. In this sense, we can generalize the concepts of Near-MDS and Almost-MDS to convolutional codes, see for instance [19].

Definition 1. We say that a convolutional $\mathcal{C}$ code is $\alpha$-AlmostMDP if $\Lambda_{j}^{1} \leq \alpha$ for all $j \leq L$ and $\alpha$ is minimal with this property.

Definition 2. We say a convolutional code is an s-Near MDP code if $\Lambda_{j}^{s}=0$ for every $j=0,1, \ldots, L$ and $s$ is minimal with this property.

The construction of MDP convolutional codes is closely related to the construction of the so-called lower triangular 
Toeplitz superregular matrices [15]. This class of matrices are characterized by having all of its "nontrivial" minors different from zero. Unfortunately, constructions of these matrices, and consequently of MDP convolutional codes, over small fields are unknown (although it was conjectured that such constructions exist, see [10], [15], [16], [17], [21]). Hence, it is reasonable to consider the norm

$$
\sum_{\substack{i \leq k \\ j \leq L}} \Lambda_{j}^{i}
$$

which we call the overall defect of the code.

To look for codes for which $\boldsymbol{\Lambda}$ is "small" is a more feasible approach, as we can see in the following example.

Example 1. Consider the convolutional code with parameters $n=4, k=2$ and $\delta=2$ over $\mathbb{F}_{3}$ whose generator matrix is

$$
G(D)=\left[\begin{array}{cccc}
1 & 0 & 1+2 D & 2+D \\
0 & 1 & 2+D & 2+D
\end{array}\right]
$$

and

$$
G_{0}=\left[\begin{array}{llll}
1 & 0 & 1 & 2 \\
0 & 1 & 2 & 2
\end{array}\right], \quad G_{1}=\left[\begin{array}{llll}
0 & 0 & 2 & 1 \\
0 & 0 & 1 & 1
\end{array}\right] .
$$

For $j=0$, we have that $d_{0}^{1}=3$ and $d_{0}^{2}=4$ (both attain the bound). Consider now the truncated generator matrix for $j=1$ :

$$
G_{1}^{\mathcal{C}}=\left[\begin{array}{cc:cc:c:ccc}
1 & 0 & 1 & 2 & O & 2 & 1 \\
0 & 1 & 2 & 2 & O & 1 & 1 \\
\hdashline & 0 & & 1 & 0 & 1 & 2 \\
\hdashline & & & 0 & 1 & 2 & 2
\end{array}\right]
$$

The matrix

$$
\left[\begin{array}{cc:cc}
1 & 2 & 2 & 1 \\
2 & 2 & 1 & 1 \\
\hdashline O & -1 & 1 & 2 \\
\hdashline & & 2 & 2
\end{array}\right]
$$

is not superregular, so the code will not be MDP [16], [17].

In this case, the truncated codeword obtained taking the sum of the first two rows is given by

$$
\left[\begin{array}{llllllll}
1 & 1 & 0 & 1 & 0 & 0 & 0 & 2
\end{array}\right]
$$

and has weight 4 . Therefore, $d_{1}^{1}=4$, which does not attain the bound $\Omega_{1}^{1}=5$. It is also possible to check that $d_{1}^{2}=\Omega_{1}^{2}=6$.

As $L=\lfloor\delta / k\rfloor+\lfloor\delta /(n-k)\rfloor=2$, we calculate the matrix

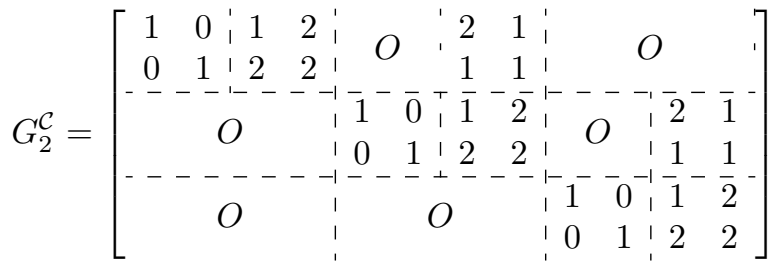

In this case, the corresponding generalized column distances are given by: $d_{2}^{1}=\Omega_{2}^{1}-3=4, d_{2}^{2}=\Omega_{2}^{2}-2=6$. The corresponding defect matrix of this code is given by:

$$
\Lambda=\left[\begin{array}{ll}
0 & 0 \\
1 & 0 \\
3 & 2
\end{array}\right]
$$

The overall defect of this code is $\|\Lambda\|=6$.

We emphasize that if one wants to construct and MDP convolutional code with these parameters, one needs to construct a superregular matrix of size $7 \times 7$ (see [15], [17], [10]). In this work, we consider that superregular matrices are lower triangular matrices, and the minimum required field size necessarily to construct an MDS convolutional code (or equivalently the superregular matrix) can be found in [21]. Computer search shows that these bounds are not optimal and there exist several conjectures on the actual minimum field size required to construct MDP for a given set of parameter $(n, k, \delta)$. For the example at hand, it can be computed that the minimum field size to construct a $7 \times 7$ superregular matrix is 17 which is significantly larger that the one used in our example $\left(\mathbb{F}_{3}\right)$.

\section{CONCLUSIONS}

In this work we introduce a generalization of the Hamming weight hierarchy tailor-made to generalize the notion of column distance of convolutional codes. The results naturally extend the existing results on the GHW for block codes and convolutional codes. This new notion leads to the generalization of the concepts of Almost-MDS and Near-MDS. More characterizations of these notions and concrete constructions of convolutional codes with a design column distance profile are left for future research.

\section{ACKNOWLEDGMENT}

This work was supported by grant 2013/25977-7, São Paulo Research Foundation (FAPESP). The work of the first author was also supported by FAPESP, grant 2015/07246-0. The third author was supported by Portuguese funds through the CIDMA - Center for Research and Development in Mathematics and Applications, and the Portuguese Foundation for Science and Technology (FCT-Fundação para a Ciência e a Tecnologia), within project PEst-UID/MAT/04106/2013.

\section{REFERENCES}

[1] K. Wei, Victor, "Generalized hamming weights for linear codes," IEEE Transactions on Information Theory, vol. 37, pp. 1412-1418, 1991.

[2] T. Kasami, T. Takata, S. Fujiwara, and S. Lin, "On the optimum bit order with respect to the state complexity of trellis diagrams for binary linear codes," IEEE Transactions on Information Theory, vol. 39, no. 1, pp. 242-245, 1993.

[3] P. Vanroose, "Code construction for the noiseless binary multiple access channel," IEEE Transactions on Information Theory, vol. 34, no. 5, pp. 1100-1106, 1988

[4] F. J. MacWilliams and N. J. A. Sloane, The Theory of Error-Correcting Codes, 6th ed. Amsterdam: North-Holland, 1988.

[5] S. Roman, Coding and Information Theory. New York, NY: Springer, 1992.

[6] S. M. Dodunekov and I. N. Landgev, "On near-MDS codes," Journal of Geometry, vol. 54, pp. 30-43, 1995.

[7] M. A. De Boer, "Almost MDS codes," Designs, Codes and Cryptography, vol. 9, no. 2, pp. 143-155, 1996. 
[8] D. Forney, "Dimension/Length Profiles and Trellis Complexity of Linear Block Codes ," IEEE Transactions on Information Theory, vol. 41, no. 2 pp. 1741-1752, 1995.

[9] T. Helleseth and T. Klove and V.I. Levenshtein and O. Ytrehus, "Bounds on the Minimum Support Weights," IEEE Transactions on Information Theory, vol. 40, no. 6, pp. 432-440, 1994.

[10] V. Tomás, "Complete-MDP convolutional codes over the erasure channel," Ph.D. dissertation, Departamento de Ciencia de la Computación e Inteligencia Artificial, Universidad de Alicante, Alicante, España, July 2010.

[11] V. Tomás, J. Rosenthal, and R. Smarandache, "Decoding of MDP convolutional codes over the erasure channel," in Proceedings of the 2009 IEEE International Symposium on Information Theory (ISIT 2009). Seoul, Korea: IEEE, June 2009, pp. 556-560.

[12] L. Cruvinel Lemes and M. Firer, "Generalized weights and bounds for error probability over erasure channels," in Proceedings of 2014 Information Theory and Applications Workshop (ITA), 2014, pp. 1-8.

[13] F. Didier, "A new upper bound on the block error probability after decoding over the erasure channel," IEEE Transactions on Information Theory, vol. 52, no. 10, pp. 4496-4503, Oct 2006.

[14] S. Fashandi, S. O. Gharan, and A. K. Khandani, "Coding over an erasure channel with a large alphabet size," in 2008 IEEE International Symposium on Information Theory, July 2008, pp. 1053-1057.

[15] P. Almeida, D. Napp, and R. Pinto, "A new class of superregular matrices and MDP convolutional codes," Linear Algebra and its Applications, vol. 439, no. 7, pp. 2145-2157, 2013.

[16] P. Almeida, D. Napp, and R. Pinto, "Superregular matrices and applications to convolutional codes," Linear Algebra and its Applications, vol. 499, no. 7, pp. 1-25, 2016.

[17] H. Gluesing-Luerssen, J. Rosenthal, and R. Smarandache, "Strongly MDS convolutional codes," IEEE Transactions on Information Theory, vol. 52, no. 2, pp. 584-598, 2006.

[18] J. Rosenthal, and E. York, " On the generalized Hamming weights of convolutional codes," IEEE Transactions on Information Theory, vol. 43, no. 1, pp. 330-335, 1997.

[19] R. Dodunekova, and S.M. Dodunekov, and T. Klove, " Almost-MDS and near-MDS codes for error detection," IEEE Transactions on Information Theory, vol. 43, no. 1, pp. 285-290, 1997.

[20] R. Johannesson and K. Sh. Zigangirov. Fundamentals of Convolutional Coding. IEEE Press, New York, 1999.

[21] R. Hutchinson, and R. Smarandache, and J. Trumpf, , "On superregular matrices and MDP convolutional codes," Linear Algebra and its Applications, vol. 428, pp. 2585-2596, 2008.

[22] S. Ball, "On sets of vectors of a finite vector space in which every subset of basis size is a basis." J. Eur. Math. Soc, vol 14(3), pp. 733-748, 2010. 